\section{Berufsverband der Deutschen Urologen e.V.}

Urologe 2012 $51: 746$

DOI 10.1007/s00120-012-2900-z

๑) Springer-Verlag 2012
Redaktion

W. Bühmann,Wenningstedt/Sylt

\title{
$\#+\#$ URO-Telegramm
}

\section{Strukturreform - BDU- Hauptstadtbüro in Berlin eröffnet}

Ein weiterer Schritt der im letzten Jahr von den Mitgliedern beschlossenen Strukturreform ist umgesetzt: am 1.5. öffnete in Berlin das BDUHauptstadtbüro als Stützpunkt der Geschäftsführung nahe der Gremien der Politik, Kassenverbände und ärztlichen Selbstverwaltung. Um Synergien zu nutzen, besteht eine Bürogemeinschaft mit der "Stiftung Männergesundheit e.V." und dem "Dachverband der Prostatazentren e.V... Die Adresse lautet: Claire-Waldoff-Str.3, 10117 Berlin. Ansprechpartner für Mitgliederfragen bleibt weiterhin die BDU-Geschäftsstelle mit Frau Habeder in Düsseldorf. Hier sehen Sie ein paar Impressionen:
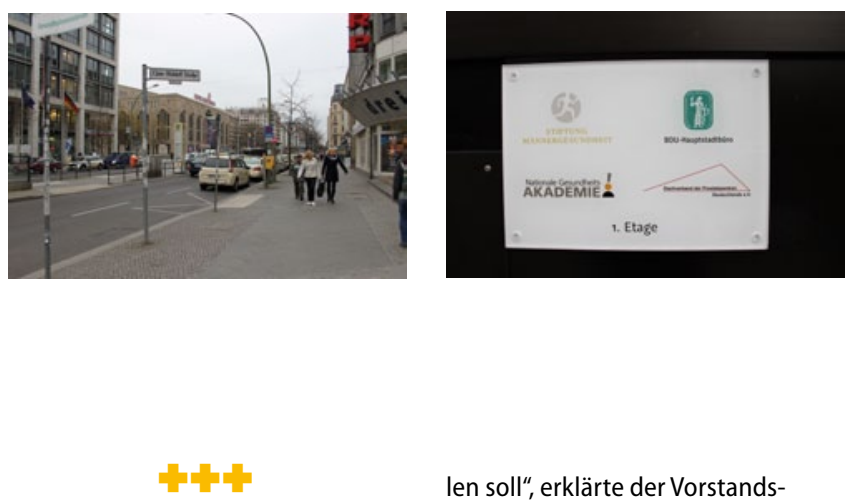

\section{KBV stellt Konzept zur Versorgung von Heimbewohnern vor}

Die medizinische Versorgung für Heimbewohner verbessern möchte die Kassenärztliche Bundesvereinigung (KBV).,"Wir haben ein Konzept erarbeitet, bei dem ein Team von Haus- und Fachärzten in Zusammenarbeit mit den Pflegekräften eine zukunftssichere und qualitativ hochwertige Versorgung in Pflegeeinrichtungen sicher $\neg$ stel-
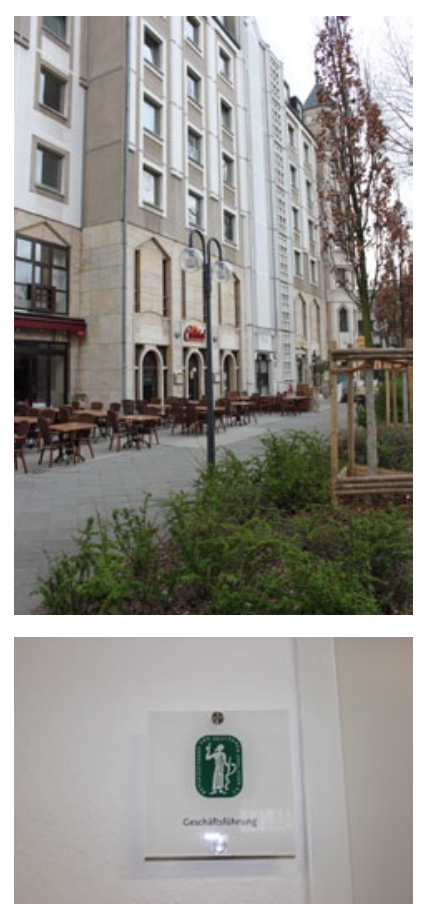

len soll", erklärte der Vorstandsvorsitzende der KBV, Andreas Köhler, bei der Vorstellung des Konzeptes heute in Berlin. Danach soll vor allem eine enge fachübergreifende Zusammenarbeit die Versorgung verbessern. Hausärzte und Fachärzte zum Beispiel für Urologie Gynäkologie, Neurologie und Nervenheilkunde sollen sich daran beteiligen. Patienten haben nach dem KBV-Konzept immer einen direkten Ansprechpartner, weil die Behandlung unter den Ärzten enger abgestimmt ist. Das führt zu einer erweiterten Visitenrufbereitschaft. Mul- timorbide Patienten, die viele verschiedene Arzneimittel einnehmen, sollen von der verbesserten Kommunikation unter den behandelnden Ärzten profitieren. Die Teambetreuung soll außerdem den Patienten in Pflegeeinrichtungen Krankenhausaufenthalte ersparen. Vorgesehen sind in dem Konzept im Bedarfsfall auch Beratungsgespräche für die Angehörigen. Köhler appellierte an die Krankenkassen, die Herausforderungen des demografischen Wandels gemeinsam zu bewältigen und wies daraufhin, dass die KBV mit dem neuen Konzept ein Ziel des geplanten Pflege-NeuausrichtungsGesetzes aufnehme. Danach soll die KBV gemeinsam mit dem Spitzenverband der gesetzlichen Krankenversicherung eine Vereinbarung zur Verbesserung der Qualität der Versorgung von Patienten in Pflegeeinrichtungen schließen.

\section{(c) hil/aerzteblatt.de}

\section{보나부}

\section{Rekordausgaben für Gesundheit}

Die Gesundheitsausgaben in Deutschland haben 2010 einen Höchstwert erreicht. Insgesamt wurden dafür 287,3 Milliarden Euro ausgegeben - pro Kopf also 3510 Euro.

Die Gesundheitsausgaben in Deutschland sind 2010 auf einen $\mathrm{Re}$ kordwert gestiegen. Insgesamt wurden dafür 287,3 Milliarden Euro ausgegeben - so viel wie nie zuvor.Im Vergleich zum Jahr 2009 nahmen die Ausgaben um 3,2 Prozent (8,9 Milliarden Euro) zu, teilte das Statistische Bundesamt in Wiesbaden zum Weltgesundheitstag mit. Rechnerisch wurden für jeden Einwohner 3510 Euro (2009: 3400 Euro) ausgegeben.Mit 165,5 Milliarden Euro trug die gesetzliche Krankenversicherung rund 58 Prozent der gesamten Gesundheitsausgaben. Den stärksten Zuwachs gab es bei der sozialen Pflegeversicherung. Deren Ausgaben nahmen um 6,0 Prozent auf 21,5 Milliarden Euro zu. Zusammengerechnet haben die Statistiker alle Gesundheitsausgaben zum Beispiel von Krankenversicherungen, Pflegeversicherungen sowie von privaten Haushalten und Organisationen mit dem Ziel der Prävention, Behandlung, Rehabilitation und Pflege. Enthalten sind auch die Verwaltungskosten und Investitionsausgaben.
Termin-Erinnerung gemeinschaft" UROLOGEN

Wer von Ihnen, liebe Kolleginnen und Kol-

leginnen, sich bisher noch nicht zu dem Seminar „Berufsausübungsgemeinschaft“ , das vom Bund der Urologen veranstaltet wird, angemeldet hat, kann dies für den Termin

am 16.6.2012 von 10- 15.30 Uhr in Köln

beim Bund der Urologen, Tel.0621-66003025,

E-mail info@bdu-eg.de, nachholen. Dort bekommen Sie auch unter www.bdu-eg.de Informationen zum Inhalt - insbesondere geht es um die Möglichkeiten, die Onkologische Versorgung für urologische Praxen zu erhalten. 